\title{
A Direct Synthesis Approach for Microwave Filters With a Complex Load and Its Application to Direct Diplexer Design
}

\author{
Ke-Li Wu, Senior Member, IEEE, and Wei Meng
}

\begin{abstract}
This paper presents a direct synthesis approach for general Chebyshev filters terminated with a complex load. The new approach is based on the fact that the polynomial functions for synthesizing the filters are composed for any matched loads. By normalizing the polynomial functions with assumed complex matched load impedance by a real reference load impedance using power waves normalization, a set of new polynomial functions for the same filter, but with real load impedance, can be formulated, from which the coupling matrix for the physical filter design can be obtained using a standard direct filter synthesis approach.

This new direct synthesis approach can find many applications. A practical application is the direct diplexer design with a realistic junction model being taken into account. With the diplexer design is concerned, a fast-converged iterative scheme is proposed. The effectiveness and the validation of the proposed scheme are demonstrated by two design examples.
\end{abstract}

Index Terms-Chebyshev filter, diplexer, filter synthesis, impedance matching.

\section{INTRODUCTION}

$\mathbf{T}$ HE SYNTHESIS of microwave filters has attracted a great deal of attention over the last few decades. The most significant work for the exact synthesis of microwave filters includes the multicoupled resonator filter network by Atia and Williams [1] and Atia et al. [2] and the direct synthesis approach for general Chebyshev filters by Cameron [3], [4].

All of these direct synthesis techniques assume that the twoport filter network is terminated by real valued reference impedances at the two ends. In fact, this reference impedance is a symbolic substitute for the characteristic impedance of the transmission line connected to the microwave filter. Generally, the two reference impedances at the two ports of a filter network, i.e., $Z_{1}$ and $Z_{2}$ in Fig. 1, can be arbitrary impedance values. However, the filters synthesized by the existing direct approaches can only

Manuscript received October 24, 2006; revised January 15, 2007. This work was supported by the Research Grants Council of the Hong Kong Special Administrative Region under Grant 2150499.

K.-L. Wu is with the Department of Electronic Engineering, The Chinese University of Hong Kong, Shatin, Hong Kong (e-mail: klwu@ee.cuhk.edu.hk).

W. Meng was with the Department of Electronic Engineering, The Chinese University of Hong Kong, Shatin, Hong Kong. He is now with the Department of Electrical and Computer Engineering, University of Maryland at College Park, College Park, MD 20742 USA.

Color versions of one or more of the figures in this paper are available online at http://ieeexplore.ieee.org.

Digital Object Identifier 10.1109/TMTT.2007.895175

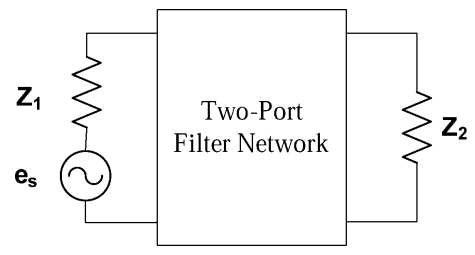

Fig. 1. Filter network terminated with reference impedances $Z_{1}$ and $Z_{2}$.

be used for the applications where the two ports of a filter are interfaced to the same reference impedance.

In many applications, a filter network is used in the circumstance in which the reference impedance at one end is a complex valued impedance, whereas the other end of the filter is terminated by a real valued reference impedance. Such complex valued impedance could be the input impedance of an antenna prefixed to the filter or the output impedance of a power amplifier suffixed to the filter in a front-end system. In the scenario of a diplexer design, the complex impedance would be the input impedance looking into the junction of the diplexer where the channel filters are attached to. However, the issue of how to directly synthesize a general Chebyshev filter with a complex reference impedance has never been systematically addressed.

Synthesis of a diplexer is a classic subject in the community and has led to a large amount of literature. An early work on direct synthesis techniques can be found in the paper by Haine and Rhodes [5] published in the 1970s. The effort has been continued by many researchers, such as in [6]-[8] and a very recent work [9]. An investigation on how to optimize the performance of a diplexer using a symmetric junction was also performed [10]. The major limitations in [5]-[9] include: 1) the junction is modeled by a simple series resistance or a shunt reactance, which has a large variation from the actual junction and 2) the parameters for the two channel filters are initially derived at the same working frequency. Very recently, people has attempted to incorporate the actual effect of the junction into the channel filter design by modifying the first irises and cavities next to the junction of the two channel filters to compensate for the complex impedance effect of the junction [11].

On the other hand, the diplexer design could start from an actual junction model and two channel filters that are separately designed. Nonlinear optimization is then used to find a satisfactory overall performance of a diplexer [12]-[14]. Although the optimization approaches are considered to be practical and can give reasonable results for most of cases if the junction meets the required conditions and the initial starting point is lucky enough, 


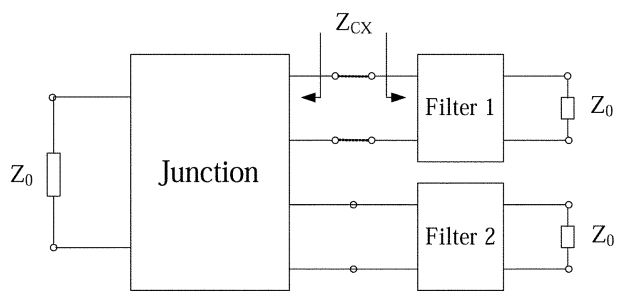

Fig. 2. Schematic diagram of a diplexer; all ports are terminated by the matched reference impedances.

people have never stopped seeking an efficient, systematic, and mathematically elegant direct technique for synthesizing microwave diplexers. A more systematic design technique that can take into account the actual junction effect is desirable.

This paper will firstly propose a new concept for direct synthesis of a general Chebyshev microwave filter with a complex load as the reference impedance. The concept is based on the fact that the rational polynomial functions that define the reflection and transfer functions of a filter are virtually referenced to two matched impedances of any value. Using the theory of power waves normalization [15], the rational functions with an assumed matched complex load can be normalized by real valued loads at the both filter ends so that the existing direct synthesis techniques for general Chebyshev filters, such as [3], can still be employed. In other words, all the existing direct approaches can be extended to the cases in which the filter is terminated by a complex load at one end and a real valued load on the other.

As depicted in Fig. 2, when the common port of the junction is connected to the matched reference impedance $Z_{0}$ and the right-bottom port of the junction is loaded with channel filter 2 , the input impedance $Z_{\mathrm{CX}}$ looking into the above-right port of junction can be viewed as the complex load impedance at the port of channel filter 1. Via the new concept proposed in this paper, channel filter 1 can be synthesized straightforwardly. Obviously the same procedures can be applied to channel filter 2. Therefore, a diplexer with a specific junction model can be designed in an iterative manner.

Before the iterative design approach is detailed, the new concept for direct synthesis of a general Chebyshev microwave filter with a complex load will be formulized. The new concept is justified by two diplexer design examples: a coaxial diplexer with a simple wire Y-junction and a waveguide diplexer using an $E$-plane T-junction with a coupled slit, respectively.

\section{Synthesis of A Filter With a COMPLEX LOAD}

The starting point for the direct synthesis of generalized Chebyshev filter is to construct three polynomials, i.e., $E(s), F(s)$, and $P(s)$, using a well developed procedure with pre-described transmission zeros (TZs), a reflection level, and the order of the filter [3]. The transfer function $S_{21}$ and the reflection functions $S_{11}$ are then defined from these polynomials. It is worth mentioning that the terminating conditions to evaluate the transfer and reflection functions do not stipulate any specific impedance value. Therefore, a filter with equiripple responses can be interpreted to be with a complex reference impedance at one end and a real reference impedance at the other end.

The main idea of this new concept is to normalize the assumed complex matched impedance at one end of the filter to the same real valued matched impedance as that at the other end of the filter. Being aware of that, the evaluation of transfer and reflection functions depends on the terminal conditions [15], three new polynomials $E^{\prime}(s), F^{\prime}(s)$, and $P^{\prime}(s)$ for real valued terminations can be formulated from those with a complex termination and the complex load. It is obvious that the responses of a filter with two real valued load impedances correspond to the measurement of a standalone filter.

\section{A. Renormalization of Reference Impedances}

Suppose that the scattering matrix $[S]$ of a two-port network is given and is referenced by the matched impedance $Z_{1}$ at port 1 and $Z_{2}$ at port 2, as shown in Fig. 1.

If the terminal impedances of the two-port network are changed from $Z_{i}$ to $Z_{i}^{\prime}(i=1$ and 2$)$, the new scattering matrix $\left[S^{\prime}\right]$ can be expressed in terms of the original $[S]$ and the reflection coefficient $r_{i}$ of $Z_{i}^{\prime}$ with respect to $Z_{i}^{*}[15]$ as

$$
\left[S^{\prime}\right]=[A]^{-1}\left([S]-[\Gamma]^{+}\right)([I]-[\Gamma][S])^{-1}[A]^{+}
$$

where $[I]$ is the identity matrix, $[\Gamma]$ and $[A]$ are the diagonal matrices with their $i$ th diagonal elements being

$$
\begin{aligned}
& \Gamma_{i}=r_{i}=\frac{Z_{i}^{\prime}-Z_{i}}{Z_{i}^{\prime}+Z_{i}^{*}} \\
& A_{i}=\frac{1-r_{i}^{*}}{\left|1-r_{i}\right|} \sqrt{\left|1-r_{i} r_{i}^{*}\right|}
\end{aligned}
$$

and the superscript " -1 " and ${ }^{+}$represent the matrix inverse and matrix complex conjugate transpose, respectively.

Considering that

$$
\begin{aligned}
\left([S]-[\Gamma]^{+}\right) & =\left[\begin{array}{ll}
S_{11} & S_{12} \\
S_{21} & S_{22}
\end{array}\right]-\left[\begin{array}{cc}
r_{1}^{*} & 0 \\
0 & r_{2}^{*}
\end{array}\right] \\
& =\left[\begin{array}{cc}
S_{11}-r_{1}^{*} & S_{12} \\
S_{21} & S_{22}-r_{2}^{*}
\end{array}\right] \\
([I]-[\Gamma][S])^{-1} & =\left(\left[\begin{array}{cc}
1-r_{1} S_{11} & -r_{1} S_{12} \\
-r_{2} S_{21} & 1-r_{2} S_{22}
\end{array}\right]\right)^{-1} \\
& =\left[\begin{array}{cc}
\frac{1-r_{2} S_{22}}{\Delta_{S}} & \frac{r_{1} S_{12}}{\Delta_{S}} \\
\frac{r_{2} S_{21}}{\Delta_{S}} & \frac{1-r_{1} S_{11}}{\Delta_{S}}
\end{array}\right]
\end{aligned}
$$

where

$$
\Delta_{S}=\left(1-r_{1} S_{11}\right)\left(1-r_{2} S_{22}\right)-r_{1} r_{2} S_{12} S_{21}
$$

the new scattering matrix after the change of the terminal impedances from $Z_{i}$ to $Z_{i}^{\prime}(i=1$ and 2) becomes (6), shown at the bottom of the following page.

In designing a channel filter for a diplexer, the reference impedances at two ports of a channel filter, when detached from the junction, are the same reference impedance associated to the transmission line connected to the filter. When the filter is loaded on the junction, the reference impedance at the connecting port will be amended to a complex number to reflect the 
presence of the junction. However, in this scenario, only the reference impedance at one port is changed.

Without losing generality, assuming port 2 of a filter is originally connected to a complex load and is to be changed to the same real valued impedance as that of port 1, i.e., $Z_{2}^{\prime}=Z_{1}^{\prime}=$ $Z_{1}=Z_{0}$, where $Z_{0}$ is a positive real number standing for the characteristic impedance of the transmission line connected to the ports of the filter, and $Z_{2}=Z_{\mathrm{CX}}$, which is a complex value related to the complex load, the above equations can be greatly simplified as

$$
\begin{aligned}
r_{1} & =0 \\
r_{1}^{*} & =0 \\
\Delta_{S} & =\left(1-r_{1} S_{11}\right)\left(1-r_{2} S_{22}\right)-r_{1} r_{2} S_{12} S_{21} \\
& =1-r_{2} S_{22} \\
S_{11}^{\prime} & =S_{11}+\frac{S_{12} r_{2} S_{21}}{\left(1-r_{2} S_{22}\right)} \\
& =\frac{S_{11}-r_{2} S_{11} S_{22}+r_{2} S_{12} S_{21}}{\left(1-r_{2} S_{22}\right)} \\
S_{12}^{\prime} & =\frac{\sqrt{\left|\operatorname{Re} Z_{2}^{\prime}\right|}}{\sqrt{\left|\operatorname{Re} Z_{2}\right|}}\left(1-r_{2}\right) \frac{S_{12}}{1-r_{2} S_{22}} \\
S_{21}^{\prime} & =\frac{\sqrt{\left|\operatorname{Re} Z_{2}^{\prime}\right|}}{\sqrt{\left|\operatorname{Re} Z_{2}\right|}}\left(1-r_{2}\right) \frac{S_{21}}{1-r_{2} S_{22}} \\
S_{22}^{\prime} & =\frac{\left(1-r_{2}\right)}{\left(1-r_{2}^{*}\right)} \frac{S_{22}-r_{2}^{*}}{1-r_{2} S_{22}} .
\end{aligned}
$$

Since the real part of input impedance of a passive network is always positive, the following relation has been used in deriving $S_{21}^{\prime}$ in (8c):

$$
\frac{\sqrt{\left|\operatorname{Re} Z_{2}\right|}}{\sqrt{\left|\operatorname{Re} Z_{2}^{\prime}\right|}} \frac{\left(1-r_{2} r_{2}^{*}\right)}{\left(1-r_{2}^{*}\right)}=\frac{\sqrt{\left|\operatorname{Re} Z_{2}^{\prime}\right|}}{\sqrt{\left|\operatorname{Re} Z_{2}\right|}}\left(1-r_{2}\right) .
$$

\section{B. Transformation of Transfer and Reflection Functions}

It is our hypothesis that a general Chebyshev filter that is with equiripple return loss in its passband and is with a complex load is to be designed. The transfer and reflection polynomials for a matched general Chebyshev filter can be composed by an existing standard procedure [3] in the format of

$$
\begin{aligned}
S_{11} & =\frac{F_{11}(s)}{E(s)} \\
S_{22} & =\frac{F_{22}(s)}{E(s)}
\end{aligned}
$$

$$
S_{12}=S_{21}=\frac{P(s) / \varepsilon}{E(s)}
$$

where $\varepsilon$ is a normalization constant related to the prescribed return loss level. According to the procedure, for a matched Chebyshev characteristics, there is

$$
F_{11}(s)=F_{22}(s)=F(s) .
$$

It is assumed that the polynomials $E(s), F(s)$, and $P(s)$ have been normalized to their respective highest degree coefficients. Both $E(s)$ and $F(s)$ are $N$ th-degree polynomials, $N$ is the degree of the filtering function, whereas $P(s)$, which contains the finite-position prescribed TZs, is of degree $n_{\mathrm{fz}}$, where $n_{\mathrm{fz}}$ is the number of finite-position TZs. It is also noticed that the polynomials $E(s), F(s)$, and $P(s)$ are related by

$$
F(s) \cdot F(s)-P(s) / \varepsilon \cdot P(s) / \varepsilon=E(s) \cdot E^{*}(s)
$$

where the roots of polynomial $E^{*}(s)$ form mirror imaginary pairs about the imaginary axis with corresponding roots of polynomial $E(s)$, and $E(s)$ is a Hurwitz polynomial and all of its roots are located in the left half-plane of the complex $s$-plane.

Substituting (10) into (8) yields

$$
\begin{aligned}
S_{11}^{\prime}= & \frac{\frac{F(s)}{E(s)}-r_{2} \frac{F(s)}{E(s)} \frac{F(s)}{E(s)}+r_{2} \frac{P(s) / \varepsilon}{E(s)} \frac{P(s) / \varepsilon}{E(s)}}{1-r_{2} \frac{F(s)}{E(s)}} \\
= & \frac{E(s) F(s)-r_{2} E(s) E^{*}(s)}{E(s)\left(E(s)-r_{2} F(s)\right)} \\
= & \frac{F(s)-r_{2} E^{*}(s)}{E(s)-r_{2} F(s)} \\
S_{12}^{\prime}= & S_{21}^{\prime} \\
= & \frac{\sqrt{\left|\operatorname{Re} Z_{2}^{\prime}\right|}}{\sqrt{\left|\operatorname{Re} Z_{2}\right|}\left(1-r_{2}\right) \frac{P(s) / \varepsilon}{E(s)-r_{2} F(s)}} \\
S_{22}^{\prime}= & \frac{\left(1-r_{2}\right)}{\left(1-r_{2}^{*}\right)} \frac{F(s)-r_{2}^{*} E(s)}{E(s)-r_{2} F(s)} .
\end{aligned}
$$

Therefore, the new transfer and reflection polynomials, after changing the terminal impedance at port 2 from $\mathrm{Z}_{2}$ to $\mathrm{Z}_{0}$, can be transformed as

$$
\begin{aligned}
E^{\prime}(s) & =E(s)-r_{2} F(s) \\
F_{11}^{\prime}(s) & =F(s)-r_{2} E^{*}(s) \\
F_{22}^{\prime}(s) & =F(s)-r_{2}^{*} E(s)
\end{aligned}
$$

$$
\begin{aligned}
& {\left[S^{\prime}\right]=[A]^{-1}\left([S]-[\Gamma]^{+}\right)([I]-[\Gamma][S])^{-1}[A]^{+}} \\
& =\left[\begin{array}{cc}
\left(\frac{S_{11}-r_{1}^{*}}{\left(1-r_{1}^{*}\right)} \frac{1-r_{2} S_{22}}{\Delta_{S}}+\frac{S_{12}}{\left(1-r_{1}^{*}\right)} \frac{r_{2} S_{21}}{\Delta_{S}}\right)\left(1-r_{1}\right) & \frac{\sqrt{\left|\operatorname{Re} Z_{1}\right|}}{\sqrt{\left|\operatorname{Re} Z_{2}^{\prime}\right|}}\left(1-r_{2}\right)\left(\frac{S_{11}-r_{1}^{*}}{\left(1-r_{1}^{*}\right)} \frac{r_{1} S_{12}}{\Delta_{S}}+\frac{S_{12}}{\left(1-r_{1}^{*}\right)} \frac{1-r_{1} S_{11}}{\Delta_{S}}\right) \\
\frac{\sqrt{\left|\operatorname{Re} Z_{2}^{\prime}\right|}}{\sqrt{\left|\operatorname{Re} Z_{2}\right|}}\left(1-r_{1}\right)\left(\frac{S_{21}}{\left(1-r_{2}^{*}\right)} \frac{1-r_{2} S_{22}}{\Delta_{S}}+\frac{S_{22}-r_{2}^{*}}{\left(1-r_{2}^{*}\right)} \frac{r_{2} S_{21}}{\Delta_{S}}\right) & \left(\frac{S_{21}}{\left(1-r_{2}^{*}\right)} \frac{r_{1} S_{12}}{\Delta_{S}}+\frac{S_{22}-r_{2}^{*}}{\left(1-r_{2}^{*}\right)} \frac{1-r_{1} S_{11}}{\Delta_{S}}\right)\left(1-r_{2}\right)
\end{array}\right]
\end{aligned}
$$


TABLE I

5-1 Filtering FunCtion: RoOTs OF $E(s), F(s)$, AND $P(s)$ POLYNOMIALs

\begin{tabular}{c|c|c|c}
\hline & Roots of $P(s)$ & Roots of $F(s)$ & Roots of $E(s)$ \\
\hline \hline 1 & $+\mathrm{j} 1.42$ & $-\mathrm{j} 0.9375$ & $-0.2802-\mathrm{j} 1.1977$ \\
\hline 2 & -- & $-\mathrm{j} 0.4901$ & $-0.6840-\mathrm{j} 0.6070$ \\
\hline 3 & -- & $+\mathrm{j} 0.1636$ & $-0.7180+\mathrm{j} 0.2381$ \\
\hline 4 & -- & $+\mathrm{j} 0.7064$ & $-0.4269+\mathrm{j} 0.8773$ \\
\hline 5 & -- & $+\mathrm{j} 0.9695$ & $-0.1126+\mathrm{j} 1.1010$ \\
\hline & $\varepsilon=1.5479$ & & \\
\hline
\end{tabular}

\begin{tabular}{l|cccccccc|}
\multicolumn{1}{c}{} & $\mathrm{S}$ & 1 & 2 & 3 & 4 & 5 & $\mathrm{~L}$ \\
$\mathrm{~S}$ & 0 & 1.0540 & 0 & 0 & 0 & 0 & 0 \\
1 & 1.0540 & 0.0366 & 0.7544 & 0.4941 & 0 & 0 & 0 \\
2 & 0 & 0.7544 & -0.6410 & 0.5101 & 0 & 0 & 0 \\
3 & 0 & 0.4941 & 0.5101 & 0.1053 & 0.6526 & 0 & 0 \\
4 & 0 & 0 & 0 & 0.6526 & 0.0506 & 0.9018 & 0 \\
5 & 0 & 0 & 0 & 0 & 0.9018 & 0.0366 & 1.0540 \\
$\mathrm{~L}$ & 0 & 0 & 0 & 0 & 0 & 1.0540 & 0 \\
\hline
\end{tabular}

(a)

\begin{tabular}{l|cccccccc|}
\multicolumn{1}{c}{$\mathrm{S}$} & \multicolumn{1}{c}{1} & 2 & 3 & 4 & 5 & $\mathrm{~L}$ \\
$\mathrm{~S}$ & 0 & 1.0540 & 0 & 0 & 0 & 0 & 0 \\
1 & 1.0540 & 0.0366 & 0.7544 & 0.4941 & 0 & 0 & 0 \\
2 & 0 & 0.7544 & -0.6410 & 0.5101 & 0 & 0 & 0 \\
3 & 0 & 0.4941 & 0.5101 & 0.1053 & 0.6526 & 0 & 0 \\
4 & 0 & 0 & 0 & 0.6526 & 0.0506 & 0.9018 & 0 \\
5 & 0 & 0 & 0 & 0 & 0.9018 & -1.6296 & 1.6665 \\
$\mathrm{~L}$ & 0 & 0 & 0 & 0 & 0 & 1.6665 & 0 \\
\hline
\end{tabular}

(b)

Fig. 3. (a) Coupling matrix $[M]$ synthesized from polynomials $E(s), F(s)$, and $P(s)$. (b) Coupling matrix $\left[M^{\prime}\right]$ from polynomials $E^{\prime}(s), F^{\prime}(s)$, and $P^{\prime}(s)$.

and

$$
P^{\prime}(s)=P(s) .
$$

It can be shown that $E^{\prime}(s)$ is still a Hurwitz polynomial and the roots of $F_{11}^{\prime}(s)$ and $F_{22}^{\prime}(s)$ form mirror imaginary pairs as expected for an unmatched filter. In fact, (13) gives all the required polynomials for the same general Chebyshev filter that was originally terminated by one complex load and this complex load has been replaced by the same real load as that connected to the other end of the filter. Note that the complex load is evaluated at the center frequency of the filter.

\section{Illustration Example}

To illustrate the new concept, an example is given here for a fifth-degree 22-dB equiripple return-loss filter with a $\mathrm{TZ}$ positioned at $+j 1.42$ in the normalized $s$-plane to give a rejection sidelobe of $30 \mathrm{~dB}$ on the upper side of the passband.

Following the standard procedure [3], the polynomials $E(s), F(s)$, and $P(s)$ according to the filter requirement can be easily constructed. The roots of the polynomials are given in Table I.

For unit reference impedance $Z_{0}$ at two ports, the $N+2$ coupling matrix $[M]$ according to the polynomials $E(s), F(s)$, and $P(s)$ can be synthesized and is given in Fig. 3(a). Theoretically, the $S$-parameters directly derived from this coupling
TABLE II

5-1 Filtering FunCtion: RoOTs OF $E^{\prime}(s), F^{\prime}(s)$, AND $P^{\prime}(s)$ POLYNOMials

\begin{tabular}{c|c|c|c|c}
\hline & Roots of $P^{\prime}(s)$ & Roots of $F^{\prime}{ }_{l 1}(s)$ & Roots of $F^{\prime}{ }_{22}(s)$ & Roots of $E^{\prime}(s)$ \\
\hline \hline 1 & $+\mathrm{j} 1.42$ & $0.1764-\mathrm{j} 1.0092$ & $-0.1764-\mathrm{j} 1.0092$ & $-0.2477-\mathrm{j} 1.0782$ \\
\hline 2 & -- & $0.3594-\mathrm{j} 0.2709$ & $-0.3594-\mathrm{j} 0.2709$ & $-0.5484-\mathrm{j} 0.2970$ \\
\hline 3 & -- & $0.2801+\mathrm{j} 0.5760$ & $-0.2801+\mathrm{j} 0.5760$ & $-0.4199+\mathrm{j} 0.6344$ \\
\hline 4 & -- & $0.0818+\mathrm{j} 1.0133$ & $-0.0818+\mathrm{j} 1.0133$ & $-0.1082+\mathrm{j} 1.0502$ \\
\hline 5 & -- & $-2.5638+\mathrm{j} 1.7689$ & $2.5638+\mathrm{j} 1.7689$ & $-2.5639+\mathrm{j} 1.7688$ \\
\hline & $\varepsilon=1.5479$ & & & \\
\hline
\end{tabular}

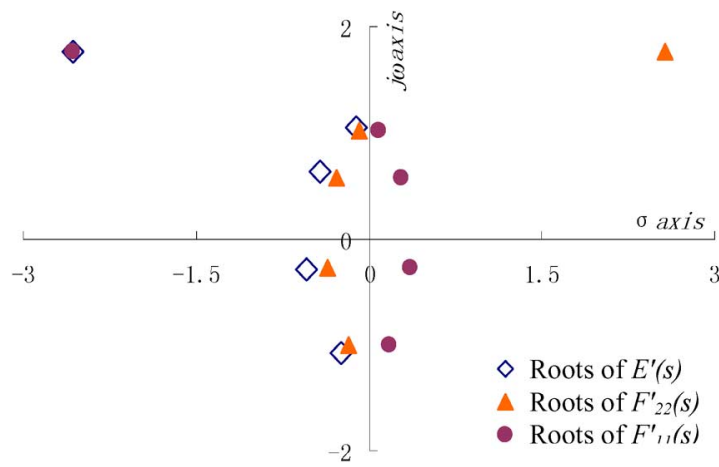

Fig. 4. Locations of roots of $E^{\prime}(s), F_{11}^{\prime}(s)$, and $F_{22}^{\prime}(s)$ on the complex $s$-plane.

matrix should give an equiripple response in the passband and a $\mathrm{TZ}$ at normalized frequency 1.42 .

In order to synthesize the coupling matrix $\left[M^{\prime}\right]$ having the same responses, but with a complex reference impedance at one port, say, at port 2, a new set of polynomials $E^{\prime}(s), F^{\prime}(s)$, and $P^{\prime}(s)$ must be sought. Without losing generality, the complex reference impedance $Z_{2}=0.4+j 0.6$ is considered in this example. By setting $Z_{2}^{\prime}$ to unit impedance $Z_{0}$, the reflection coefficient $r_{2}$ will be $r_{2}=\left(Z_{2}^{\prime}-Z_{2}\right) /\left(Z_{2}^{\prime}+Z_{2}^{*}\right)=0.5172-\mathrm{j} 0.2069$.

Using (13), the new polynomials $E^{\prime}(s), F_{11}^{\prime}(s), F_{22}^{\prime}(s)$, and $P^{\prime}(s)$ can be found, the roots of which are given in Table II. It is shown in Fig. 4 that the roots of $F_{11}^{\prime}(s)$ and $F_{22}^{\prime}(s)$ form mirror image pairs about the imaginary axis, and the roots of $E^{\prime}(s)$ still satisfy the Hurwitz condition, all of which lie in the left plane of the complex $s$-plane.

An existing coupling matrix synthesis procedure can be applied to the new polynomials to obtain a new coupling matrix $\left[M^{\prime}\right]$, as given in Fig. 3(b). Moreover, the lossless condition of a two-port network has to be guaranteed, which means that an additional coefficient must be used to adjust the $S$-parameters. In this case, a coefficient $\varepsilon_{n}=1.5811$ can be found to be multiplied to polynomial $P^{\prime}(s)$ in addition to the coefficient $\varepsilon$. Comparing the two coupling matrices in Figs. 3(a) and (b), the only changes happen on coupling values of the load coupling from the last resonator to the load $Z_{2}$ and the resonant frequency of the last resonator next to the load.

Three sets of $S$-parameters curves are superimposed in Fig. 5. The "matched" and "unmatched" cases correspond to the responses of coupling matrix $[M]$ with any matched loads and $\left[M^{\prime}\right]$ with real impedance $Z_{0}$ at the two ends of the filter, respectively. For "verification" purposes, the circuit model simulation results of coupling matrix $\left[M^{\prime}\right]$ with port 1 terminated by $Z_{0}$ and port 2 terminated by $Z_{2}=0.4+j 0.6$ are also provided in 


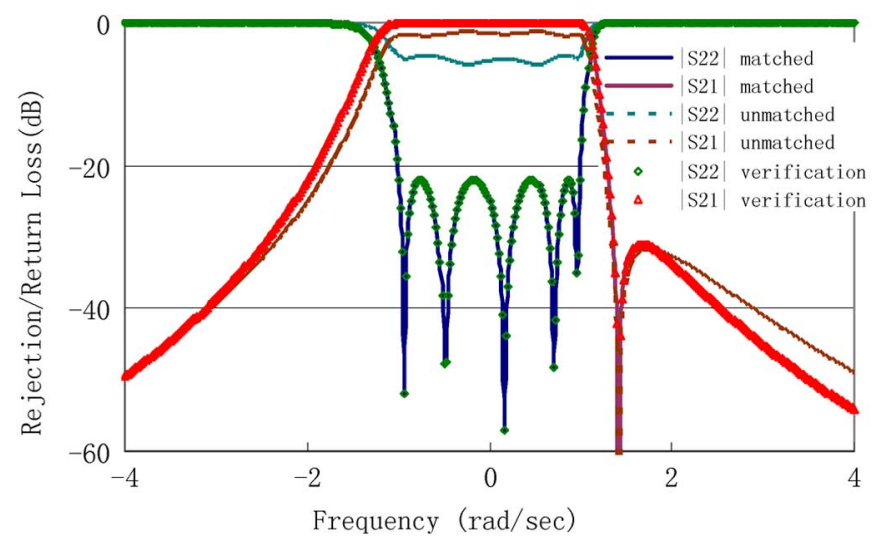

Fig. 5. Comparison of $S$-parameters for the matched, unmatched, and verification filters.

this figure. As expected, the "verification" curves are identical to those of the "matched" case.

\section{DiReCt DipleXer Design}

The most common approach to realize a diplexer is to use two bandpass filters that are combined through a three-port junction. Different types of junction configurations have been seen for various of applications. For RF coaxial diplexers for mobile phone base-station applications, a star-shaped wire Y-junction is commonly used, whereas for the microwave diplexers consisting of waveguide channel filters, a waveguide junction should be employed, commonly including $E$ - or $H$-plane waveguide $\mathrm{T}$ - or $\mathrm{Y}$-junctions. For designing broadband diplexers, one needs to optimize the performances of the junctions to satisfy certain necessary conditions, e.g., using a symmetric Y-junction [10] or a waveguide T-junction with a tuning conductor post [16].

Since the two channel filters are electrically connected to each other through a junction, the parameters of the two filters must be considered together in conjunction with the properties of the chosen junction in order to take into account the interaction among the filters and the junction. As depicted in Fig. 2, assuming that channel filter 2 of a diplexer with center frequency $f_{2}$ has been appropriately designed and the common port of the junction is matched, looking into the junction at the port where channel filter 1 is connected, one can find a load impedance $Z_{\mathrm{CX}}$ at $f_{1}$, which is the center frequency of channel filter 1 . If the reference impedance $Z_{0}$ (which corresponds to $Z_{2}^{\prime}$ ) is given and the load impedance $Z_{\mathrm{CX}}$ (which corresponds to $Z_{2}$ ) is known, the polynomials for channel filter 1 can be determined by (13). Consequently, the related coupling matrix $\left[M_{1}\right]$ for a designated filter topology can be easily obtained from the polynomials by using an existing direct filter synthesizing approach.

It will be an iterative process to design the coupling matrices for the two channel filters. Having understood how channel filter 1 is directly synthesized under the assumption that the design of channel filter 2 is converged, one can alternatively apply the same scheme to channel 2 and channel 1 until the solution for the two channel filters converged.

It needs to be pointed out that since the load impedance at the center frequency of a channel is used, the approach is, in

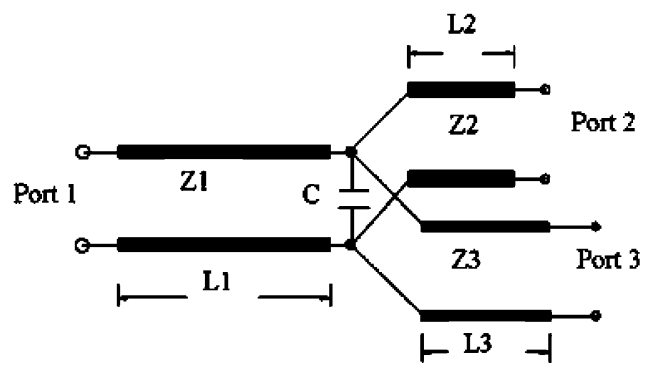

Fig. 6. Transmission line model of a wire Y-junction with a shunt capacitor.

principle, a narrowband approach. However, if the slope of the complex load with respect to frequency is small, the approach can be extended to a diplexer design with moderate bandwidth.

In the proposed design procedure, appropriate lengths for the transmission lines between the junction and the channel filters have been incorporated in the junction model. In fact, the lengths need to be carefully optimized although there are some empirical rules-of-thumb to follow. From a theoretical point-of-view, the lengths should be chosen in the way that the slopes of the complex loads at the ports facing to the two filters become minimum. How to analytically design the lengths using the proposed theory is still a future research topic.

\section{Practical Design Examples}

\section{A. Diplexer With Wire Y-Junction}

The first design example is with a wire Y-junction as the common junction connecting to two channel filters. Fig. 6 shows a generic transmission line model for a wire Y-junction with a shunt capacitor. It is assumed that port 1 of the junction is connected to the common port, and ports 2 and 3 are connected to the low and high channel filters, respectively.

The channel filters in this example are synthesized by the approach discussed in Section III. The input impedance looking into port 2 at $f_{1}$ (center frequency of the low channel filter), when a high channel filter is connected at port 3 , is, in general, a complex number. The impedance will be used as the complex load impedance in synthesizing the low channel filter. Similarly, the input impedance looking into port 3 at $f_{2}$ (center frequency of the high channel filter), when a low channel filter is connected at port 2 , is the complex load impedance in synthesizing the high channel filter. By default, port 1 is always terminated by the unit reference impedance.

To simplify the design, the characteristic impedances $Z_{1}, Z_{2}$, and $Z_{3}$ of the three sections of the transmission line in Fig. 6 are assumed to be the same, and the lengths $L_{1}, L_{2}$, and $L_{3}$ are adjustable. The two channel filters are initially designed as fifth-degree 22-dB return-loss Chebyshev filters with a TZ at $+j 1.52$ for the low channel filter and a TZ at $-j 1.52$ for the high channel filter, respectively. The center frequencies of two filters are at $f_{1}=1.74 \mathrm{GHz}$ and $f_{2}=1.85 \mathrm{GHz}$, respectively, and the bandwidth of both filters is approximately $0.08 \mathrm{GHz}$.

To begin with, the coupling matrices for the two channels with matched loads are obtained and are listed in Table III in the columns of the zeroth iteration. Connecting two channel filters 
TABLE III

COUPLING MATRICES OF LOW AND High CHANNEL FILTER IN EACH ITERATION

\begin{tabular}{|c|c|c|c|c|c|c|}
\hline & Low & Channel & & High & Channel & \\
\hline & Oth & $1 \mathrm{st}$ & $2 \mathrm{nd}$ & Oth & $1 \mathrm{st}$ & $2 \mathrm{nd}$ \\
\hline$M_{01}$ & 1.0545 & 0.9518 & 0.9063 & 1.0545 & 1.1816 & 0.9838 \\
\hline$M_{I I}$ & 0.0333 & 1.0683 & 0.8219 & -0.0333 & -1.7225 & -1.0079 \\
\hline$M_{12}$ & 0.9026 & 0.9026 & 0.9026 & 0.9026 & 0.9026 & 0.9026 \\
\hline$M_{22}$ & 0.0464 & 0.0464 & 0.0464 & $\mid-0.0464$ & -0.0464 & -0.0464 \\
\hline$M_{23}$ & 0.6525 & 0.6525 & 0.6525 & 0.6525 & 0.6525 & 0.6525 \\
\hline$M_{33}$ & 0.0982 & 0.0982 & 0.0982 & -0.0982 & -0.0982 & -0.0982 \\
\hline$M_{34}$ & 0.5361 & 0.5361 & 0.5361 & 0.5361 & 0.5361 & 0.5361 \\
\hline$M_{35}$ & 0.4495 & 0.4495 & 0.4495 & -0.4495 & -0.4495 & -0.4495 \\
\hline$M_{44}$ & -0.5865 & -0.5865 & -0.5865 & 0.5865 & 0.5865 & 0.5865 \\
\hline$M_{45}$ & 0.7827 & 0.7827 & 0.7827 & 0.7827 & 0.7827 & 0.7827 \\
\hline$M_{55}$ & 0.0333 & 0.0333 & 0.0333 & -0.0333 & -0.0333 & -0.0333 \\
\hline$M_{56}$ & 1.0545 & 1.0545 & 1.0545 & $\mid 1.0545$ & 1.0545 & 1.0545 \\
\hline
\end{tabular}

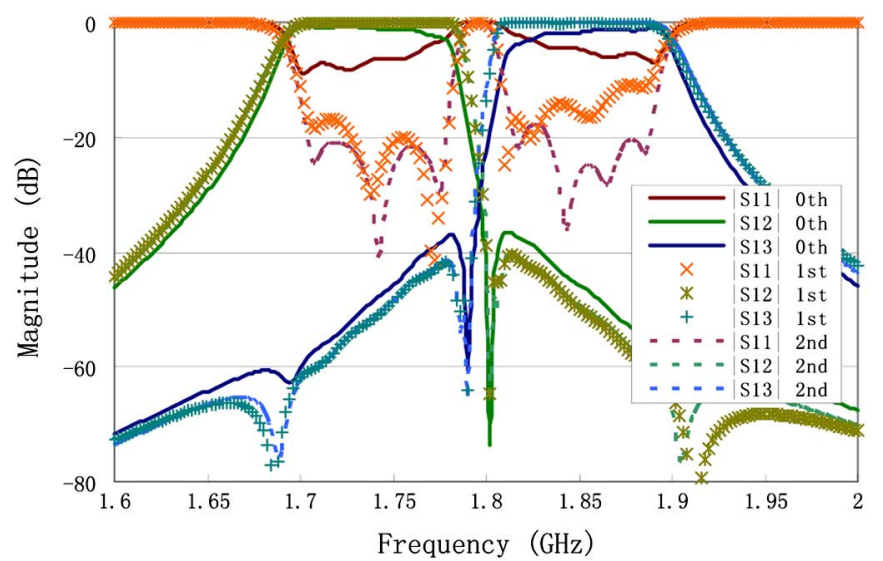

Fig. 7. Responses of the diplexer design using a wire Y-junction.

TABLE IV

INPUT IMPEDANCES AT THE PORTS OF Y-JUNCTION

\begin{tabular}{l|l|l|}
\multicolumn{1}{l}{} & \multicolumn{1}{l}{ Port 2 at $f_{1}$} & \multicolumn{1}{l}{ Port 3 at $f_{2}$} \\
\cline { 2 - 3 } 1st & $0.4365+\mathrm{j} 0.4063$ & $0.3796-\mathrm{j} 0.5767$ \\
\cline { 2 - 3 } 2nd & $0.4915+\mathrm{j} 0.3486$ & $0.4923-\mathrm{j} 0.4315$ \\
\cline { 2 - 3 } & &
\end{tabular}

directly onto the Y-junction, the circuit response of the diplexer in this iteration is marked by "0th" and is shown in Fig. 7.

Table IV lists the input impedances looking into ports 2 and 3 , while the channel filter at the other channel that is designed in the previous iteration is connected. The coupling matrices directly synthesized for each iteration are given in Table III. The circuit responses of the diplexer for each iteration are superimposed in Fig. 7. It should be mentioned that the diplexer design reaches its satisfactory converged response only by three iterations. Notice that the responses in Fig. 7 are simulated based on the circuit model of the Y-junction and the circuit model of channel filters. A possible realization of this diplexer in a coaxial combline structure is proposed in Fig. 8.

\section{B. Diplexer Design Using an E-Plane T-Junction}

The performance of a waveguide T-junction is of great importance in designing a waveguide diplexer. The standard $\mathrm{T}$-junction is widely used for narrowband diplexers. In practice, additional adjusting elements, such as coupled slit [14], inductive post [16], and reflection stub [12], [13] are required to

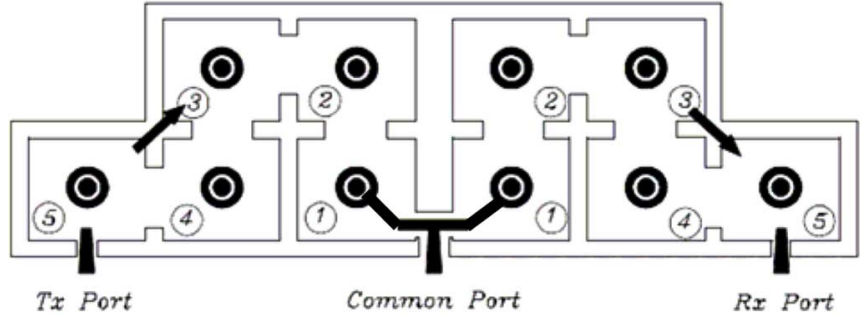

Fig. 8. Coaxial combline diplexer with two five-pole channel filters.

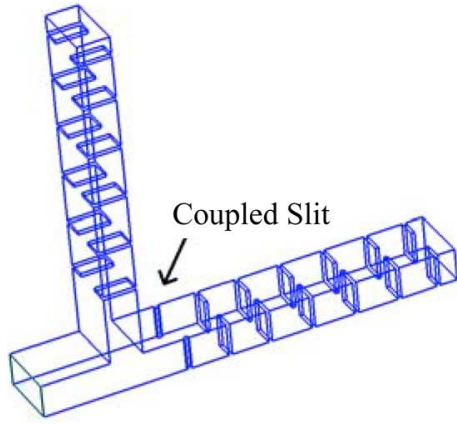

Fig. 9. Waveguide diplexer using an $E$-plane T-junction with a coupled slit.

TABLE V

INPUT IMPEDANCES AT THE PORTS OF E-PLANE T-JUNCTION

\begin{tabular}{l|l|l|}
\multicolumn{1}{c}{} & \multicolumn{1}{l}{ Port 2 at $f_{1}$} & \multicolumn{1}{l}{ Port 3 at f } \\
\cline { 2 - 3 } 1st & $0.2909+0.1072 \mathrm{i}$ & $0.7474-0.1072 \mathrm{i}$ \\
\cline { 2 - 3 } 2nd & $0.5916+0.0667 \mathrm{i}$ & $0.7823+0.1771 \mathrm{i}$ \\
\cline { 2 - 3 } 3rd & $0.5888+0.0523 \mathrm{i}$ & $0.7585+0.1838 \mathrm{i}$ \\
\cline { 2 - 3 } & &
\end{tabular}

TABLE VI

CHANGed COUPLING Elements OF CHANNEl FILTERS IN EACH ITERATION

\begin{tabular}{l|l|l|l|l|}
\multicolumn{5}{c}{ Low Channel } \\
\cline { 2 - 5 }$M_{01}$ & 0 th & 1 st & 2nd & 3rd \\
\cline { 2 - 5 }$M_{11}$ & 1.0570 & $\mathbf{0 . 6 0 7 6}$ & $\mathbf{0 . 8 1 8 2}$ & $\mathbf{0 . 8 1 4 3}$ \\
\cline { 2 - 5 } & 0.000 & $\mathbf{0 . 4 1 1 7}$ & $\mathbf{0 . 1 2 6 0}$ & $\mathbf{0 . 0 9 9 2}$ \\
\cline { 2 - 5 }$M_{01}$ & 1.0570 & $\mathbf{0 . 9 2 3 2}$ & $\mathbf{0 . 9 5 8 6}$ & $\mathbf{0 . 9 4 7 2}$ \\
\cline { 2 - 5 }$M_{11}$ & 0.000 & $\mathbf{- 0 . 1 6 0 3}$ & $\mathbf{0 . 2 5 2 9}$ & $\mathbf{0 . 2 7 0 7}$ \\
\cline { 2 - 5 } & &
\end{tabular}

minimize the slope of the reflection with respect to frequency over the frequencies of the channel filters. This example will demonstrate a waveguide diplexer designed using a slit-coupled $E$-plane T-junction with a bandwidth of $600 \mathrm{MHz}$.

Fig. 9 shows the waveguide diplexer using an $E$-plane T-junction with a coupled slit. The two channel filters are required to be fifth-degree 22-dB return-loss pure Chebyshev filters with two channel center frequencies at $f_{1}=12.6 \mathrm{GHz}$ and $f_{2}=14.2 \mathrm{GHz}$, respectively. The bandwidth of both filters is approximately $600 \mathrm{MHz}$. The diplexer specifications were used for a communication satellite. A WR 75 waveguide is used as the interface.

A similar design process as that for the first design example is carried out. Again, only three iterations are required. The complex load impedances in each iteration for each channel filter are summarized in Table V. Only the changed coupling elements for the filters are given in Table VI. The circuit model responses of the diplexer in each iteration are shown in Fig. 10. 


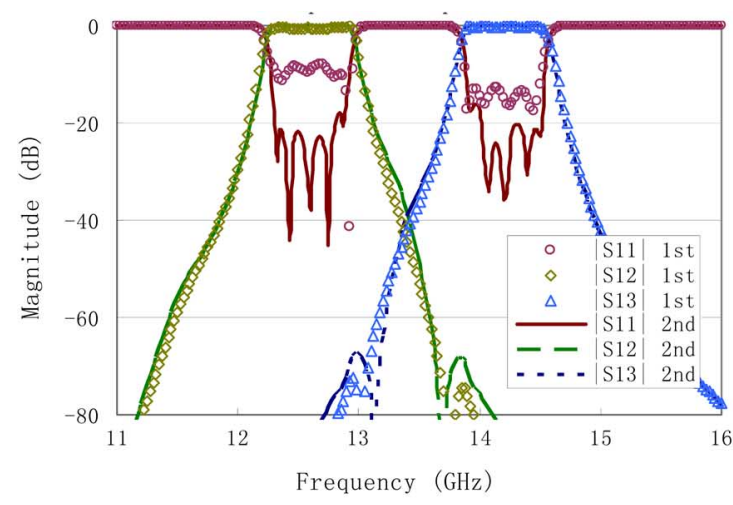

(a)

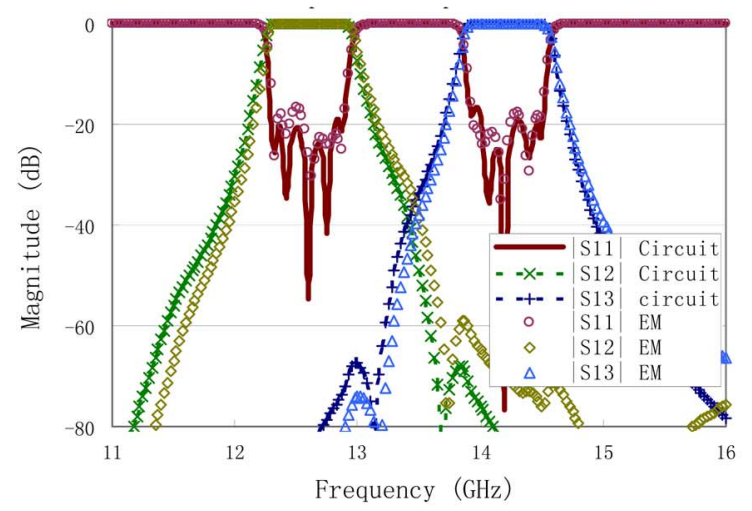

(b)

Fig. 10. Responses of the diplexer using $E$-plane T-junction. (a) First iteration and second iteration. (b) Circuit simulation (third iteration) and EM simulation.

It is noted that the simulation of the diplexer is based on the electromagnetic (EM) mode-matching (MM) T-junction model and the circuit channel filter model. The circuit model of the channel filters is converted into the physical model with physical dimensions by an MM program that has been fully verified for communication satellite payload applications. The complete physical model of the designed diplexer has also been simulated by the EM-based MM program. It is anticipated that the EM model will reflect the dispersion effects of the waveguide filters outside of the passband. An excellent agreement between the responses of the designed circuit model and the EM model can be observed.

\section{CONCLUSION}

A direct synthesis approach for microwave filters with a complex load impedance at one end and a real load impedance at the other end has been presented. A new set of filter design polynomials for the synthesizing required coupling matrix can be obtained by the power waves impedance normalization. The standard direct filter synthesis theory can be applied to the modified polynomials to derive the filter coupling matrix. The capability to incorporate a complex load impedance in the design of general Chebyshev filters makes this approach very useful to many applications. The approach can particularly be used for designing a diplexer with a realistic junction model. A novel iterative scheme for direct synthesizing the channel filters has also been proposed. The validation of the proposed scheme is firmly made through two design examples.

\section{REFERENCES}

[1] A. E. Atia and A. E. Williams, "Narrow-bandpass waveguide filters," IEEE Trans. Microw. Theory Tech., vol. MTT-20, no. 4, pp. 258-265, Apr. 1972.

[2] A. E. Atia, A. E. Williams, and R. W. Newcomb, "Narrow-band multiple-coupled cavity synthesis," IEEE Trans. Circuits Syst., vol. CAS-21, no. 9, pp. 649-655, Sep. 1974.

[3] R. J. Cameron, "General coupling matrix synthesis methods for Chebyshev filtering functions," IEEE Trans. Microw. Theory Tech., vol. 47, no. 4, pp. 433-442, Apr. 1999.

[4] — , "Advanced coupling matrix synthesis techniques for microwave filters," IEEE Trans. Microw. Theory Tech., vol. 51, no. 1, pp. 1-10, Jan. 2003.

[5] J. L. Haine and J. D. Rhodes, "Direct design formulas for asymmetric bandpass channel diplexer," IEEE Trans. Microw. Theory Tech., vol. MTT-25, no. 10, pp. 807-814, Oct. 1977.

[6] J. D. Rhodes and R. Levy, "A generalized multiplexer theory," IEEE Trans. Microw. Theory Tech., vol. MTT-27, no. 2, pp. 99-111, Feb. 1979.

[7] — "Design of general manifold multiplexer," IEEE Trans. Microw. Theory Tech., vol. MTT-27, no. 2, pp. 111-123, Feb. 1979.

[8] R. Levy, "Synthesis of non-contiguous diplexers using broadband matching theory," in IEEE MTT-S Int. Microw. Symp. Dig., Boston, MA, Jun. 10-14, 1991, pp. 543-546.

[9] G. Macchiarella and S. Tamiazzo, "Synthesis of diplexers based on the evaluation of suitable characteristic polynomials," in IEEE MTT-S Int. Microw. Symp. Dig., San Francisco, CA, Jun. 2006, pp. 111-114.

[10] A. Morini and T. Rozzi, "Constrains to the optimum performance and bandwidth limitations of diplexers employing symmetric three-port junctions," IEEE Trans. Microw. Theory Tech., vol. 44, no. 2, pp. 242-248, Feb. 1996.

[11] A. Morini, T. Rozzi, M. Farina, and G. Venanzoni, "A new look at the practical design of compact diplexers," IEEE Trans. Microw. Theory Tech., vol. 54, no. 9, pp. 3515-3520, Sep. 2006.

[12] H.-W. Yao, A. E. Abdelmonem, J.-F. Liang, X.-P. Liang, and K. A. Zaki, "Wide-band waveguide and ridge waveguide T-junctions for diplexer applications," IEEE Trans. Microw. Theory Tech., vol. 41, no. 12, pp. 2166-2173, Dec. 1993.

[13] Y. Rong, H.-W. Yao, K. A. Zaki, and T. Dolan, "Millimeter-wave $K a$-band $H$-plane diplexers and multiplexers," IEEE Trans. Microw. Theory Tech., vol. 47, no. 12, pp. 2325-2330, Dec. 1999.

[14] J. Dittloff and F. Arndt, "Computer-aided design of slit-coupled $H$-plane T-junction diplexers with $E$-plane metal-insert filters," IEEE Trans. Microw. Theory Tech., vol. 36, no. 12, pp. 1833-1840, Dec. 1988.

[15] K. Kurokawa, "Power waves and the scattering matrix," IEEE Trans. Microw. Theory Tech., vol. MTT-13, no. 3, pp. 194-202, Mar. 1965.

[16] K.-L. Wu and H. Wang, "A rigorous modal analysis of $H$-plane waveguide T-junction loaded with a partial-height post for wideband applications," IEEE Trans. Microw. Theory Tech., vol. 49, no. 5, pp. 893-901, May 2001.

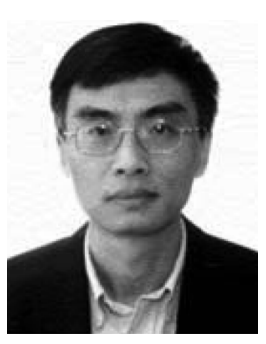

Ke-Li Wu (M'90-SM'96) received the B.S. and M.Eng. degrees from Nanjing University of Science and Technology, Nanjing, China, in 1982 and 1985, respectively, and the Ph.D. degree from Laval University, Quebec, QC, Canada, in 1989.

From 1989 to 1993 , he was with the Communications Research Laboratory, McMaster University, Hamilton, ON, Canada, as a Research Engineer and a Research Group Manager. In March 1993, he joined the Corporate Research and Development Division, Com Dev International, where he was a Principal Member of Technical Staff in charge of developing advanced electromagnetic (EM) design software for passive microwave subsystems for communication satellites. Since October 1999, he has been with the Department of Electronic Engineering, The Chinese University of Hong Kong, Shatin, Hong Kong, where he is currently a Professor. He has authored or coauthored 
numerous publications in the areas of EM modeling, microwave, and antenna engineering. His current research interests include numerical and analytical methods in electromagnetics, passive microwave circuits, filters, antennas for communication systems, low-temperature co-fired ceramic (LTCC)-based multichip modules (MCMs) for wireless communications, and RF identification (RFID) technologies.

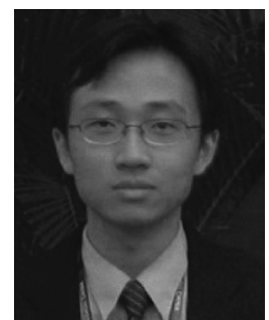

Wei Meng received the B.Eng. degree (with first-class honors) and M.Phil. degree in electronic engineering from The Chinese University of Hong Kong, Shatin, Hong Kong, in 2003 and 2006, respectively, and is currently working toward the Ph.D. degree in electrical and computer engineering at the University of Maryland at College Park.

His research is focused on the modeling and design of passive microwave components, especially on the synthesis and design techniques of microwave filters. 